Communications in Physics, Vol. 30, No. 1 (2020), pp. 11-18

DOI:10.15625/0868-3166/30/1/14582

\title{
EFFECT OF POLYPYRROLE ON THE ELECTRICAL, DIELECTRIC AND MECHANICAL PROPERTIES OF WATERBORNE EPOXY COATINGS
}

\author{
THAI THU THUY, NGUYEN THUY DUONG, NGUYEN THI THU TRANG, \\ PHAM GIA VU, TRAN DAI LAM AND NGUYEN ANH SON ${ }^{\dagger}$
}

Institute for Tropical Technology, Vietnam Academy of Science and Technology

18 Hoang Quoc Viet, Cau Giay District, Hanoi, Vietnam

${ }^{\dagger} E$-mail: nason@itt.vast.vn

Received 4 November 2019

Accepted for publication 2 December 2019

Published 28 February 2020

\begin{abstract}
In this context, dissipative composite based on waterborne epoxy system and polypyrrole (PPy) was investigated. PPy was synthesized by chemical oxidation polymerization. Its morphology and chemical structure were confirmed by using field emission scanning electron microscopy (FESEM) and Fourier transform infrared spectroscopy (FTIR). Then, PPy was welldispersed in epoxy coating and had a good compatibility with the matrix. The effects of PPy on dielectric, electrical and mechanical properties of epoxy/PPy composites were examined. The dielectric constant and electrical conductivity of coatings increased with addition of PPy fillers. Over $15 \mathrm{wt}$.\% PPy loading, the volume resistivity of samples slightly decreased from $6.7 \times 10^{10}$ to $1.5 \times 10^{10} \Omega \mathrm{cm}$. In contrast, the presence of PPy diminished both impact and abrasion resistance of the epoxy/PPy composites, down to $160 \mathrm{~kg} \mathrm{~cm}$ and $10.2 \mathrm{~L} / \mathrm{mil}$, respectively, but they stayed acceptable for the coatings. The results reveal that the epoxy containing PPy is suitable for various electrical and dielectric applications.
\end{abstract}

Keywords: polypyrrole, waterborne epoxy, dielectric, volume resistivity, impact strength, abrasion resistance.

Classification numbers: 81.40.pq; 83.80.-k; 77.84.Lf.

\section{INTRODUCTION}

In recent years, the polymer composites of an insulating polymer incorporated conducting additives have attracted plenty of attention. The new materials can present desired properties (C)2020 Vietnam Academy of Science and Technology 
for various electrical and electromagnetic applications to avoid electrostatic charges and electromagnetic aggression phenomena $[1,2]$. Commonly used conducting fillers to make insulating matrix conductive are carbon black (CB), carbon nanotube (CNT), graphene, metallic powders, flakes and intrinsically conductive polymers (ICPs) [3-7]. Due to the good electrical conductivity, lightweight and facile synthesis, the conducting polymers replace step by step the organic and expensive inorganic fillers. PPy is one of the most intensively studied ICPs. In addition to the outstanding characteristics of ICPs, it displays the environmental/chemical stability [8], and its electrical conductivity can be controlled by doping method $[9,10]$. However, in literature, some works reported that the PPy is very brittle and cannot carry any mechanical load [11].

Epoxy is well-known a thermosetting resin having excellent mechanical, thermal properties, good adhesion and chemical resistance. Combining PPy with epoxy resin is a good strategy to resolve the poor mechanical properties of PPy. Moreover, the obtained composite can present a better dielectric and electrical properties than that of neat epoxy. Zhang et al. reported that the nanofibers PPy incorporated into epoxy resins give a higher tensile strength than that of neat epoxy (90.36 MPa compared to $70.03 \mathrm{MPa}$ ) [12]. Furthermore, the presence of PPy conducted a better electrical conductivity (about $3 \times 10^{12} \Omega \mathrm{cm}$ ) and real permittivity of the epoxy matrix. Besides, the epoxy coatings containing PPy introduced also remarkable acid, corrosion and weathering resistance [13-15]. The composites of PPy with an epoxy system were also examined to replace metal compound in the isotropically conductive adhesives. The PPy doped dodecylbenzenesulphonic acid (DBSA) improved the electrical conductivity of epoxy resin up to $10^{-3} \mathrm{~S} / \mathrm{cm}$ at 15 wt.\% content with negligible effect of moisture and acceptable impact properties [16]. Today, for safety, health and environmental regulations, the solvent-free epoxy resin has more and more been required. However, there were a few works in literature that employed on the composites of PPy and these epoxy systems.

The aim of this paper is to attempt to display the effect of PPy on the properties of waterborne epoxy coatings. The PPy particles were synthesized with an oxidation polymerization method with ferric chloride $\left(\mathrm{FeCl}_{3}\right)$ as oxidant. Then, the waterborne epoxy coatings containing PPy with varying concentration were prepared. The effects of PPy on the morphology, dielectric, electrical and mechanical properties of epoxy matrix were examined by using field emission scanning electron microscope (FESEM), complex dielectric permittivity measurement, I-V method, impact and abrasion resistance.

\section{EXPERIMENT}

\section{II.1. Materials}

The coating was a commercial bicomponent water-based epoxy system. The base was a bisphenol A epoxy resin (Epikote 828, Hexion, Thailand) that has equivalent weight of about 184-190 g/eq. A water-reducible amine adducts (Epikure 8537-wy-60, Momentive, Thailand) consisted of 60 wt.\% solids in water, 2-propoxyethanol and glacial acetic acid was used as a hardener which has an amine value of $310-360 \mathrm{mg} / \mathrm{g}$ and an equivalent weight of $174 \mathrm{~g} / \mathrm{eg}$.

Pyrrole (Py, $\geq 97 \%$ ) and reagent ferric chloride $\left(\mathrm{FeCl}_{3}\right.$, used as an oxidizing agent) were purchased from Sigma-Aldrich. The Py monomer was purified before polymerization process. Hydrochloric acid $(\mathrm{HCl})$ and acetic acid $\left(\mathrm{CH}_{3} \mathrm{COOH}\right)$ were provided by Merck and used as received. 
In this study, the metallic substrates were plates of carbon steel XC35 with size of $150 \times$ $100 \times 1 \mathrm{~mm}$. They were polished with $\mathrm{SiC}$ papers (400 and 600 grades) and cleaned with distilled water and ethanol.

\section{II.2. Sample preparation}

PPy was synthesized by chemical oxidation polymerization as described elsewhere [16]. Briefly, fresh Py was added in a $1 \mathrm{M} \mathrm{HCl}$ solution and stirred for 30 mins. The oxidant $\left(\mathrm{FeCl}_{3}\right)$ was dissolved in another $1 \mathrm{M} \mathrm{HCl}$ solution with stoichiometry $\mathrm{Py} / \mathrm{FeCl}_{3}$ ratio $(1 / 2.33)$, then it was poured slowly drop-wise in the Py solution. The polymerization process was kept at room temperature for $24 \mathrm{~h}$. The obtained black precipitation was filtered and washed several times with distilled water to remove all residuals. The produces were dried at $80^{\circ} \mathrm{C}$ overnight.

For the composite coatings, the synthesized PPy was incorporated into epoxy system with various concentrations: 10, 15, 20, 25 and 30 wt.\%, henceforth called Epo-PPy10, Epo-PPy15, Epo-PPy20, Epo-PPy25 and Epo-PPy30, respectively. To obtain a well-dispersion of PPy in epoxy matrix, in all cases, the conducting polymer was added in the hardener solution (water was adjusted to receive a mixture with similar viscosity for all samples). These mixtures were then strongly stirred by magnetic stirring and ultrasonicated by T10 IKA disperser, Ultra-Turrax for $4 \mathrm{~h}$. The obtained suspensions were added epoxy resin with stoichiometry ratio into and stirred for $1 \mathrm{~h}$. The epoxy/PPy composite coatings were applied onto the surface of carbon steel substrate by spin coating technique. After drying a week in air, the samples were $25-30 \mu \mathrm{m}$ thick.

\section{II.3. Characterization}

Field emission scanning electron microscope (FESEM) was used to observe the morphology of synthesized PPy and epoxy/PPy composite coatings. The FESEM images were recorded at $20 \mathrm{kV}$ for powder and $2 \mathrm{kV}$ for coatings. A thin layer of platinum had been sputtered onto all samples. The chemical structure of synthesized PPy was characterized by Fourier-transform infrared (FTIR) spectra by using a Nexus 670 Nicolet spectrometer with a resolution of $4 \mathrm{~cm}^{-1}$ in the range from 400 to $4000 \mathrm{~cm}^{-1}$.

The complex dielectric constant of the epoxy/PPy composites were determined in the frequency ranged from $1.43 \mathrm{MHz}$ to $1000 \mathrm{~Hz}$ with $1 \mathrm{~V}$ AC voltage on an Agilent E4980A instrument equipment with 16451B test figure. The volume resistivity of coatings was measured by I-V method according to ASTM D257.

The impact strength of epoxy/PPy composite coatings was determined according to ASTM D2794. The values of impact resistance were obtained with maximum height of standard weight $(2 \mathrm{~kg}$ ) dropped on the surface of samples, and no fracture were observed at the contact zone. The abrasion resistance of samples was measured by Falling Sand method according to ASTM D968. The coated substrates were set up at an angle of $45^{\circ}$, then the sand falls from the height of $90 \mathrm{~cm}$ onto the surface of samples. The tests were conducted in increment of $1 \mathrm{~L}$ of sand.

\section{RESULTS AND DISCUSSION}

Fig. 1 shows the FESEM image of synthesized PPy. It illustrates the typical spherical morphology of PPy with size of about $800 \mathrm{~nm}$ and porosity [17]. The PPy particles were agglomerated to form larger globules with the granules overlapped over each other, which looks like the morphology observed on carbon black [18] due to the existence of an attractive force between the 
individual polymer chains. So, depending on the interaction of polymer chains, the size of PPy agglomeration can present various levels.

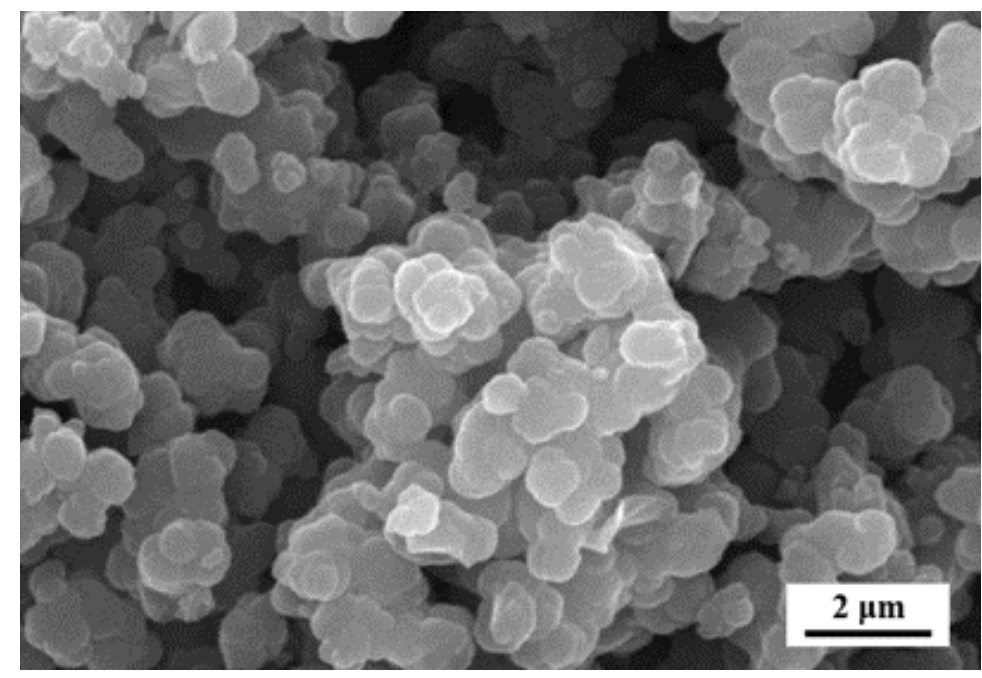

Fig. 1. FESEM micrograph of synthesized PPy.

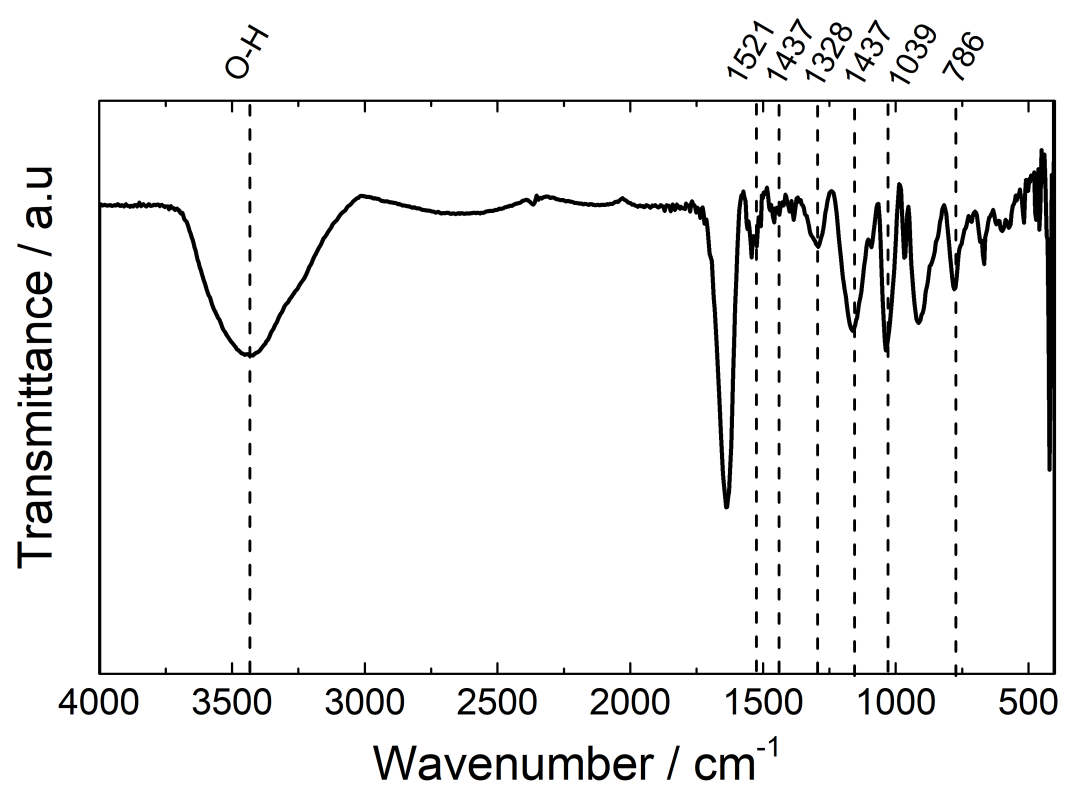

Fig. 2. FTIR spectra of synthesized PPy.

To confirm the chemical structure of synthesized PPy, the FTIR spectroscopy was performed. The FTIR spectrum of synthesized PPy is shown in Fig. 2. It displays the characteristic 
bands for PPy as reported in the literature [19]. The peaks at 1521 and $1437 \mathrm{~cm}^{-1}$ are attributed to $\mathrm{C}=\mathrm{C}$ stretching and conjugated $\mathrm{C}-\mathrm{N}$ stretching in the PPy ring, respectively. The absorption bands at 1328,1154 and $1039 \mathrm{~cm}^{-1}$ are assigned to $\mathrm{C}-\mathrm{H}$ bending, $\mathrm{C}-\mathrm{H}$ in-plane stretching and $\mathrm{C}-\mathrm{H}$ vibration of 2,5-substituted Py, respectively [20]. Furthermore, the FTIR spectrum also shows a vibration band at $786 \mathrm{~cm}^{-1}$ belonging to the $\mathrm{C}-\mathrm{H}$ wagging vibration.

The morphology of epoxy composite coatings containing PPy with different proportions was then investigated. To observe the compatibility and the dispersibility of the PPy in the waterborne epoxy matrix, the FESEM micrographs were recorded at the cross-section of the films (Fig. 3). For the neat epoxy coatings, the analyzed area is smooth and uniform as expected (Fig. 3a). At the fracture section of coatings containing PPy (Fig. 3b-f), it appears many broken marks in not the same direction as seen in the case of neat epoxy coating. The micrographs reveal a good dispersion of PPy in the epoxy matrix. As seen in Fig. 3, the broken marks are more and more crowded with the increase of PPy content in the epoxy system. In addition, it cannot be observed the phase separation between the PPy spheres and the matrix for all coatings containing the PPy indicating a good compatibility of two polymers.
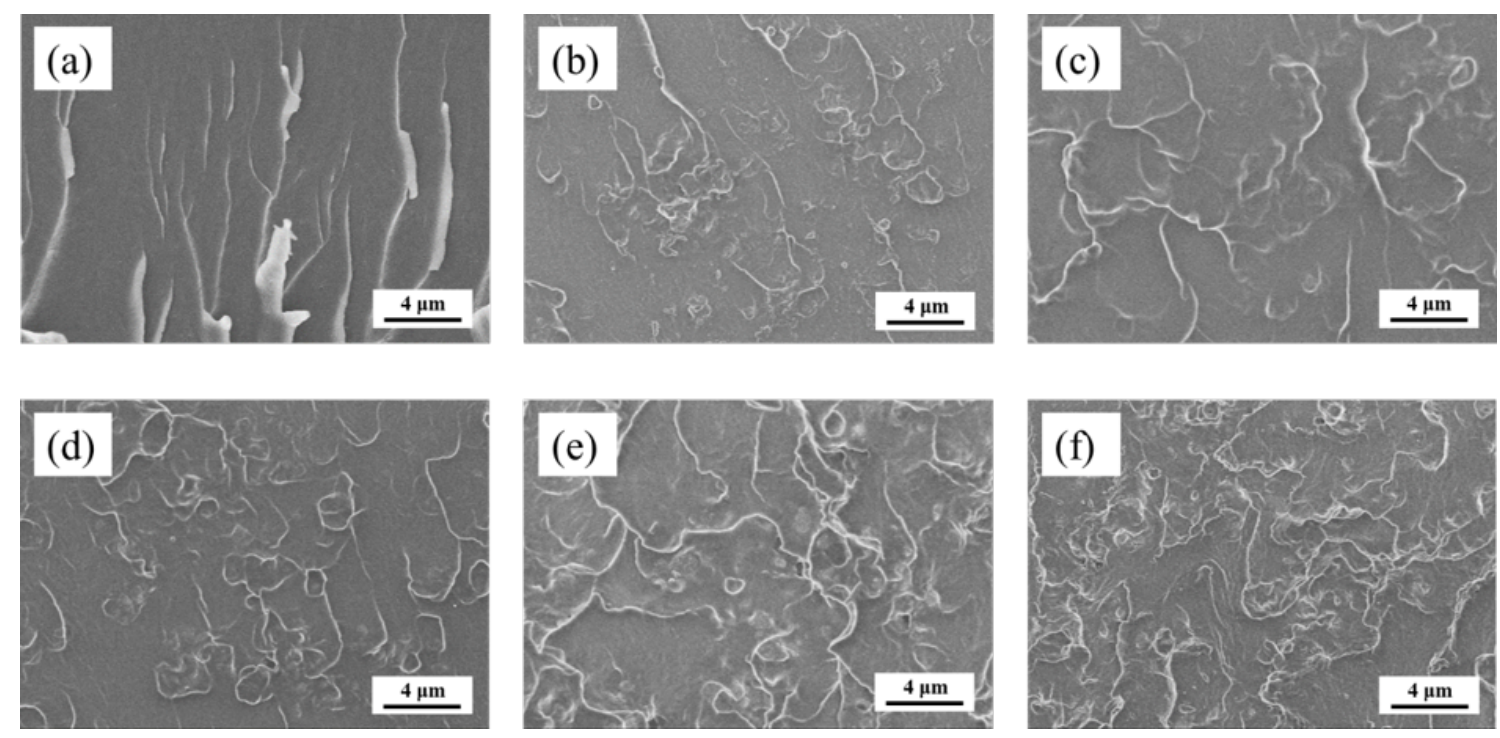

Fig. 3. FESEM micrographs at cross-section of epoxy (a); Epo-PPy10 (b); Epo-PPy15 (c); Epo-PPy20 (d); Epo-PPy25 (e) and Epo-PPy30 (f).

Fig. 4 displays the dielectric permittivity complex of composites via the variation of real (relative dielectric, $\varepsilon^{\prime}$ ) and imaginary (dielectric loss factor, $\varepsilon^{\prime \prime}$ ) parts as a function of frequency. As well-known insulating material, the neat epoxy shows the lowest value of dielectric constant and loss factor in the analyzed frequency range $(1000 \mathrm{~Hz}-1.4 \mathrm{MHz}), \varepsilon^{\prime}=3.2$ and $\varepsilon^{\prime \prime}=0.1$ at $10 \mathrm{kHz}$. By adding PPy powder to the epoxy matrix, the relative dielectric of composites increased to high value, up to 18.7 at $1000 \mathrm{~Hz}$ and 14.3 at $10 \mathrm{kHz}$ (for Ep-PPy30). This behavior reveals that the system exhibits a strong interfacial polarization [21]. For all coatings, the value dielectric permittivity complex increased with decreasing frequency. This behavior is due to the reorientation of dipolar groups which can easily orient themselves at lower frequency, but hardly 
orient themselves at higher frequency [22]. On the other hand, at lower frequency (around 10 $\mathrm{kHz}$ ), a plateau is observed for all epoxy/PPy composites due to the contribution of direct current (DC) process.
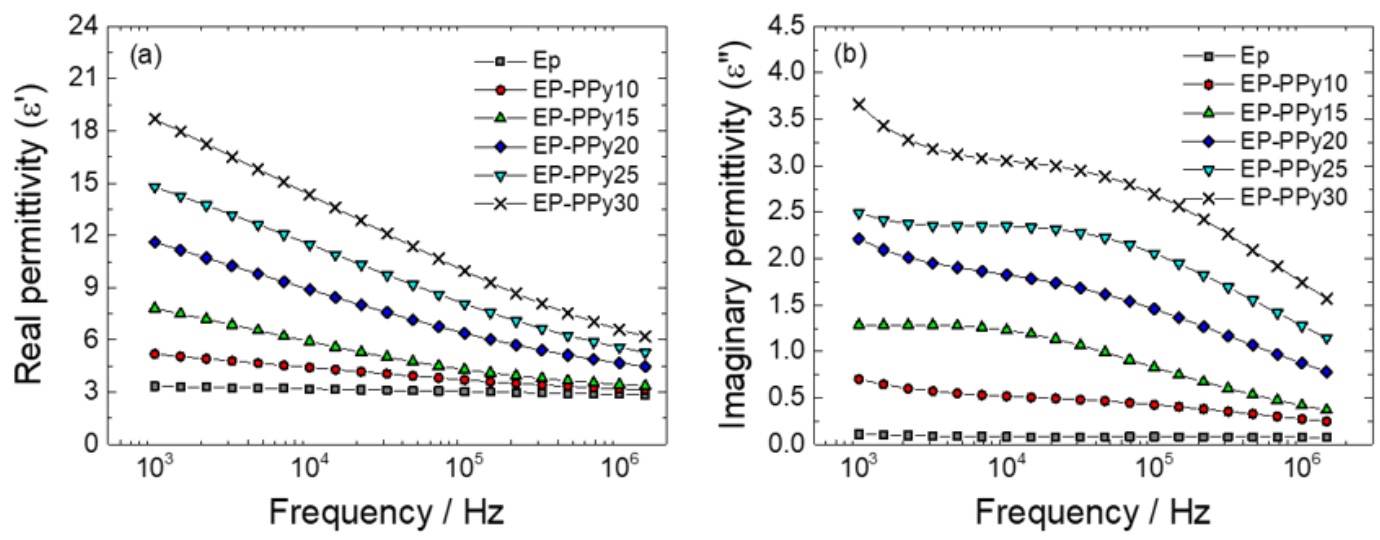

Fig. 4. Real part (a) and imaginary part (b) of complex permittivity versus applied electrical frequency for the coatings containing different PPy contents (indicated in the figure), compared to neat epoxy coating.

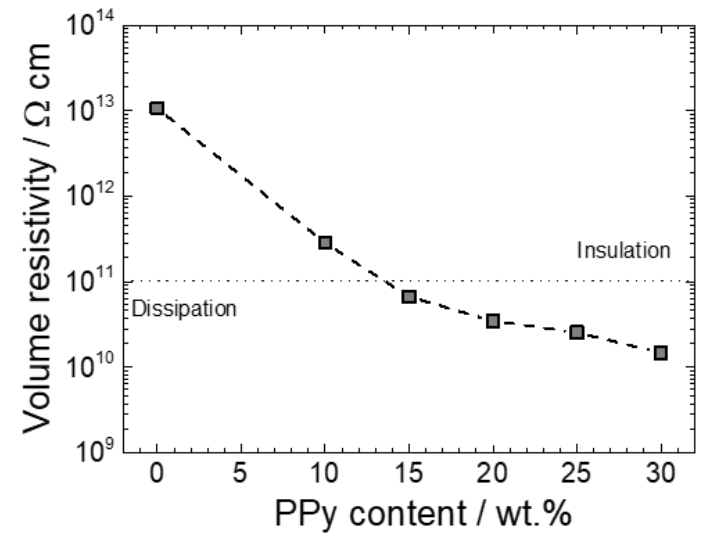

Fig. 5. Volume resistivity of epoxy/PPy composites coatings as a function of PPy contents in the epoxy system.

The DC electrical conductivity of the samples was measured by using I-V method. Fig. 5 shows the variation of volume resistivity as a function of PPy loading. The neat epoxy as insulating material has very high volume resistivity, $1.1 \times 10^{13} \Omega \mathrm{cm}$. For epoxy composites containing conducting polymer, the value of resistivity decreased with increasing the PPy contents. Between 0 and $15 \mathrm{wt} . \%$ PPy loading, the conductivity of composites epoxy/PPy is strongly enhanced, the resistivity decreased from $1.1 \times 10^{13}$ to $6.7 \times 10^{10} \Omega \mathrm{cm}$. The samples with $15 \mathrm{wt} . \%$ PPy loading 
became a dissipated material (according to ANSI/ESD S541-2003). Over this content of PPy, the resistivity of composites slightly decreases with increase of loading PPy, from $6.7 \times 10^{10}$ to $1.5 \times 10^{10} \Omega \mathrm{cm}$ (corresponding to $30 \mathrm{wt} . \%$ PPy loading). It can be explained that, at the content lower than $15 \mathrm{wt} . \%$, the PPy particles were well dispersed and filled in the epoxy matrix, but after that, they began to be saturated in the system. However, as seen in Fig. 3d, e, f, it has no important agglomeration of PPy detected in the cross-section of epoxy containing from 20 to $30 \mathrm{wt} \%$ PPy loading composites.

Fig. 6 displays the effects of PPy loading on the mechanical properties of waterborne epoxy system. It shows that, up to $15 \mathrm{wt} . \%$ PPy loading, the composites have excellent impact resistance with measured value of $200 \mathrm{~kg} \mathrm{~cm}$. With the higher PPy loading in the matrix, the impact resistance properties of epoxy/PPy composites slightly decreased to $160 \mathrm{~kg} \mathrm{~cm}$, corresponding to Epo-PPy30. Despite PPy is a brittle material [11], the impact resistance of epoxy/PPy composites stayed high. It can be explained by the insertion and/or penetration of epoxy resin into the PPy structure (overlapped sphere with high porosity) that reduce not much the impact resistance of epoxy system. However, Fig. 6 shows a decrease of abrasion resistance with the increase of PPy loading for the epoxy/PPy composites, from 16.2 to $10.1 \mathrm{~L} / \mathrm{mil}$. At $15 \mathrm{wt} \%$ PPy loading, the composite presents an abrasion resistance of $12.7 \mathrm{~L} / \mathrm{mil}, 22 \%$ lower than that of neat epoxy.

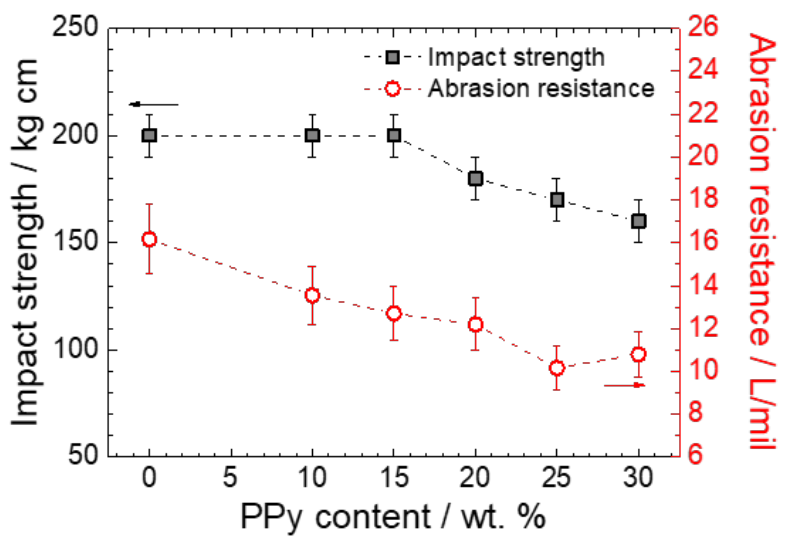

Fig. 6. Impact strength and abrasion resistance of epoxy/PPy composite coatings as a function of PPy content in the epoxy system.

The results demonstrate that in spite of the good compatibility between PPy and epoxy matrix, the interaction at interface of two components is not as good as the reticular network of epoxy. We can conclude that the increase of PPy content in the epoxy systems can enhance the electrical properties of the insulating epoxy, but it reduces the mechanical properties of the composites, especially epoxy/PPy composites with over 15 wt.\% of PPy loading.

\section{CONCLUSIONS}

In this work, the effects of PPy particles on the dielectric, electrical and mechanical properties of the waterborne epoxy coating were studied. The homogenous dispersion and compatibility of PPy in the epoxy resin with different amounts were demonstrated by FESEM. The dielectric 
constant increased with addition of PPy loading, and it was high at low frequency. It can reach up to 18.7 for Epo-PPy30 at $1000 \mathrm{~Hz}$. With over 15 wt.\% PPy content, the epoxy/PPy composite became dissipative materials with the volume resistivity down to $1.5 \times 10^{10} \Omega \mathrm{cm}$. It was found that the impact resistance of the composites slightly decreased with the presence of PPy in the composition. Below to $20 \mathrm{wt} . \%$ PPy loading, the coatings had excellent impact strength $(200 \mathrm{~kg}$ $\mathrm{cm}$ ), but with $30 \mathrm{wt} . \%$ PPy loading, the Epo-PPy30 could reach only $160 \mathrm{~kg} \mathrm{~cm}$. However, the abrasion resistance of epoxy/PPy coatings decreased with increasing of PPy content, down to 10.2 $\mathrm{L} / \mathrm{mil}$. In combination with their electrical and mechanical properties, the epoxy/PPy coatings with 15 or $20 \mathrm{wt} . \%$ of PPy loading can meet the requirements for low loss dielectric and antistatic materials.

\section{ACKNOWLEDGEMENT}

The authors gratefully acknowledge the financial support of Vietnam Academy of Science and Technology under project DLTE00.05/18-19.

\section{REFERENCES}

[1] N. Kizildag, N. Ucar, A. Onen, I. Karacan, J. Compos. Mater. 50 (28) (2016) 3981.

[2] F. Shahzad, S.H. Lee, S.M. Hong, C.M. Koo, Res. Chem. Intermediat. 44 (8) (2018) 4707.

[3] R. Schueler, J. Petermann, K. Schulte, H.-P. Wentzel, J. Appl. Polym. Sci. 63 (13) (1997) 1741.

[4] Y.J. Kim, T.S. Shin, H.D. Choi, J.H. Kwon, Y.-C. Chung, H.G. Yoon, Carbon 43 (2005) 23.

[5] H. Kim, Y. Miura, C.W. Macosko, Chem. Mater. 22 (2010) 3441.

[6] W.J. Kim, M. Taya, M.N. Nguyen, Mech. Mater. 41 (2019) 1116.

[7] H. Deligoz, B. Tieke, Macromol. Mater. Eng. 291 (2006) 793.

[8] A. Eftekhari, Synth. Met. 142 (2004) 305.

[9] M.-K. Song, Y.-T. Kim, B.-S. Kim, J. Kim, K. Char, H.-W. Rhee, Synth. Met. 141 (2004) 315.

[10] P.Dutta, S.K. De, Synth. Met. 139 (2003) 201.

[11] A.F. Diaz, B. Hall, IBM J. Res. Dev. 27 (4) (1983) 342.

[12] X. Zhang, Z. Yan, J. Guo, Z. Liu, D. Jiang, Q. He, H. Wei, H. Gu, H.A. Colorado, X. Zhang, S. Wei, Z. Guo, J. Mater. Chem. C 3 (2015) 162.

[13] S. Abhijit, A.M. Prakash, V.A. Bambole, Pigm. Resin Technol. 42 (5) (2013) 317.

[14] T.L.H. Nguyen, A.T. Trinh, Vietnam J. Chem. 46 (4) (2008) 462.

[15] N.T. Thom, T.M.T. Dinh, T.N. Pham, T.P. Nguyen, T.H.V. Vu, Q.M. Ngo, J. Electron. Mater. 48 (6) (2019) 3931.

[16] I.A. Mir, D. Kumar, J. Adhesion 86 (4) (2010) 447.

[17] C. Cassignol, M. Cavarero, A. Boudet, A. Ricard, Polymer 40 (1999) 1139.

[18] F. Gubbels, S. Blacher, E. Vanlathem, R. Jerome. R. Deltour, F. Bouers, Ph. Teyssie, Macromolecules 28 (1995) 1559.

[19] M.D. Migahed, T. Fahmy, M. Ishra, A. Barakat, Polym. Test. 23 (2004) 361.

[20] P. Dallas, D. Niarchos, D. Vrbanic, N. Boukos, S. Pejovnik, C. Trapalis, D. Petridis, Polymer 48 (7) (2007) 2007.

[21] C.-D. Liu, S.-N. Lee, C.-H. Ho, J.-L. Han, K.-H. Hsieh, J. Phys. Chem. C 112 (41) (2008) 15956.

[22] M. Rahman, C.-W. Hsieh, C.-T. Wang, B.-R. Jian, W. Lee, Dyes Pigm. 84 (2010) 128. 